\title{
Radiation pressure instability as a variability mechanism in the microquasar GRS 1915+105
}

\author{
A. Janiuk and B. Czerny \\ Nicolaus Copernicus Astronomical Center, Bartycka 18, 00-716 Warsaw, Poland \\ agnes@camk.edu.pl; bcz@camk.edu.pl \\ A. Siemiginowska \\ Harvard Smithsonian Center for Astrophysics, 60 Garden Street, MS-70, Cambridge MA 02138 \\ aneta@head-cfa.harvard.edu
}

\begin{abstract}
Physical mechanism responsible for high viscosity in accretion disks is still under debate. Parameterization of the viscous stress as $\alpha P$ proved to be a successful representation of this mechanism in the outer parts of the disk, explaining the dwarf novae and X-ray novae outbursts as due to ionization instability. We show that this parameterization can be also adopted in the innermost part of the disk where the adoption of the $\alpha$-viscosity law implies the presence of the instability in the radiation pressure dominated region. We study the time evolution of such disks. We show that the time-dependent behavior of GRS 1915+105 can be well reproduced if $\alpha$-viscosity disk model is calculated accurately (with proper numerical coefficients in vertically averaged equations and with advection included), and if the model is supplemented with (i) moderate corona dissipating $50 \%$ of energy (ii) jet carrying luminosity-dependent fraction of energy. These necessary modifications in the form of the presence of a corona and a jet are well justified observationally. The model predicts outbursts at luminosity larger than $0.16 \dot{M}_{E d d}$, as required, correct outburst timescales and amplitudes, including the effect of increasing outburst timescale with mean luminosity. This result strongly suggests that the $\alpha$-viscosity law is a good description of the actual mechanism responsible for angular momentum transfer also in the innermost, radiation pressure dominated part of the disk around a black hole.
\end{abstract}

Subject headings: accretion, accretion disks — binaries: close — black hole physics — instabilities stars: individual (GRS 1915+105)

\section{INTRODUCTION}

A standard, geometrically thin accretion disk (Shakura \& Sunyaev, 1973) is known to be unstable in the innermost, radiation pressure dominated regions (Lightman \& Eardley 1974, Pringle, Rees \& Pacholczyk 1974)). The presence of this instability is due to an assumption that the viscous torque is proportional to the total (gas+radiation) pressure. Since our understanding of the nature of viscosity in a disk is poor (for a review, see Papaloizou \& Lin 1995) we can explore the viscosity mechanism indirectly, through modeling the consequences of the radiation pressure instability and comparing them to the observed variability of $\mathrm{X}$ ray sources.

On the theoretical side, the basic description of stationary solutions was completed by Abramowicz et al. (1988) who found that radial advection stabilize the disk at high accretion rates so a time dependent behavior was expected only for intermediate accretion rates. The disk evolution was first computed by Honma, Matsuoto \& Kato (1991), and Szuszkiewicz \& Miller (1998) pre- 
sented computations of several consecutive outbursts, thus confirming a limit cycle behavior of the disk.

On the observational side, a perfect candidate has been found to study the details of radiation pressure instability. This is the X-ray source GRS 1915+105 discovered by GRANAT observatory (Castro-Tirado, Brandt \& Lundt 1992) and extensively observed by the Rossi X-Ray Timing Explorer (Belloni et al. 1997a, 1997b). The source exhibits a superluminal jet (Mirabel \& Rodriguez 1994). During outbursts, its X-ray luminosity is dominated by a disk-like component (Belloni et al 1997a), and therefore the observed variability in this state should reflect the time behavior of the disk. The variations are well modeled phenomenologically by periodic changes of the disc inner radius (e.g. Feroci et al. 1999). The scenario, in which the radiation pressure instability is actually responsible for these variations was outlined by Belloni et al. (1997c). The duration of the hard spectral state episodes in the disk-dominated epochs was recently identified with the viscous timescale of a standard, radiation pressure dominated disk (Trudolyubov, Churazov \& Gilfanov 1999).

However, the time evolution computations of Honma et al. (1991) and Szuszkiewicz \& Miller (1998) based on radiation pressure instability strongly overpredict the amplitude of the outburst of GRS 1915+105. Therefore, Nayakshin, Rapaport \& Melia (2000) suggested that the standard viscosity law does not apply and they successfully reproduced the overall shape of GRS $1915+105$ RXTE lightcurve under an assumption of viscosity law of Taam, Chen \& Swank (1997). In order to reproduce the observed minimum luminosity required for outburst behavior they also had to assume that the disk dissipates only $10 \%$ of the energy, with the remaining energy dissipated in a hot corona, which is not supported by observations.

In this paper we apply the standard viscosity assumption and nevertheless we successfully reproduce the RXTE ligthcurve of GRS 1915+105. The key elements of our model, in comparison with Honma et al. (1991) and Szuszkiewicz \& Miller (1998), are: (i) the presence of the outflow in a form of a jet (modeled after Nayakshin et al. 2000), (ii) the use of the appropriate coefficients in vertically averaged equations determined from the disk vertical structure, (iii) the presence of a moderately strong corona, dissipating $50 \%$ of the energy.

\section{METHOD}

\subsection{Assumptions}

We study the time-dependent structure of a Keplerian vertically averaged accretion disk.

We use a standard viscosity prescription, i.e. we assume that the viscous stress tensor is proportional to the total pressure, $P\left(\tau_{r \varphi}=-\alpha P\right)$.

The angular momentum distribution of disk material is approximated as Keplerian so we neglect the problem of the transonic character of the flow close to the marginally stable orbit studied by Honma et al. (1991) and Szuszkiewicz \& Miller (1998). Instead, we pay much more attention to various disk cooling mechanisms.

We assume that the heat generated within the disk at any radius is either stored temporarily within this radius or is removed by: (i) radiation (ii) advection (iii) jet. Because the spectral observations show the presence of a hard X-ray tail, we also assume the existence of a hot corona.

The radiative cooling of the disk is calculated assuming that the disk is optically thick and radiates as a black body. The fraction of energy carried by advection is determined by standard equations (e.g. Paczyński \& Bisnovatyi-Kogan 1981, Muchotrzeb \& Paczyński 1982, Abramowicz et al. 1988).

Fraction of energy, $f_{j e t}$, carried locally by a jet is parameterized after Nayakshin et al. (2000):

$$
f_{j e t}=1-\frac{1}{1+A \dot{m}^{2}} .
$$

Here the constant A is a model parameter describing the strength of the jet and $\dot{m}$ is the local accretion rate at a given moment and radius, measured in units of the Eddington accretion rate:

$$
\dot{M}_{E d d}=\frac{L_{E d d}}{c^{2} \eta}=\frac{4 \pi G M m_{H}}{\sigma_{T} c \eta} .
$$

The assumed efficiency of accretion is $\eta=1 / 16$, as it results from the pseudo-Newtonian approximation to disk accretion. 
In our model this cooling mechanism is included in the energy balance, in opposite to the model of Nayakshin et al. (2000) where the jet was only used as an energy channel carrying a fraction of energy dissipated in the corona.

The fraction of the energy dissipated in the corona is a free parameter of the model and in the present model we assume it takes a constant value, $f_{\text {cor }}=0.5$, independent from time and radius.

The time evolution of the disk is governed by two equations: energy balance given in the following form

$$
\Sigma T \frac{\partial S}{\partial t}=F_{g e n}-F_{r a d}-F_{a d v}-F_{j e t}-F_{c o r}
$$

and the standard equation describing the time evolution of the disk surface density

$$
\frac{\partial \Sigma}{\partial t}=\frac{1}{r} \frac{\partial}{\partial r}\left(3 r^{1 / 2} \frac{\partial}{\partial r}\left(r^{1 / 2} \nu \Sigma\right)\right)
$$

where the cooling by jet and corona is defined as

$$
\begin{gathered}
f_{j e t}=\frac{F_{j e t}}{F_{r a d}+F_{a d v}+F_{j e t}}, \\
f_{c o r}=\frac{F_{c o r}}{F_{r a d}+F_{a d v}+F_{j e t}+F_{c o r}},
\end{gathered}
$$

Determination of algebraic relation between the quantities in Eq. 3 and 4 results from the continuity equation, hydrostatic equilibrium and radiative transfer. It involves the determination of dimensionless coefficients which results from replacement of the disk vertical structure with vertically averaged (or equatorial) quantities (e.g. Abramowicz et al. 1988, Honma et al. 1991).

We determine those coefficients from the study of the vertical structure of a stationary disk at $10 r_{g}$ (see e.g. Dörer et al. 1996). Apart from standard ingredients, our stationary disk model included the effect of radial advection, energy transport by convection and appropriate boundfree opacities. The numerical code used for the computations was developed from the version of Pojmański (1986) and subsequently modified by Różańska et al. (1999). The coefficients, as defined by Muchotrzeb \& Paczyński (1982) and Abramowicz et al. (1988) are: $B 1=0.8, B 3=5.0$ and $B 4=6.0$, while the above authors used $B 1=0.67, B 3=B 4=6.0$ and Honma et al. (1991) used $B 1=1.0, B 3=16.0$ and $B 4=8.0$.
The importance of the model ingredients is illustrated by the stability curve of a stationary model calculated at $10 R_{S c h w}$ for $10 M_{\odot}$ black hole mass and viscosity parameter $\alpha$ equal to 0.01 (see Figure 1).

The triangles in Fig. 1 are obtained from the solution of the disk vertical structure, and dashed line is the stability curve obtained from the vertically averaged disk equations (with appropriate coefficients). In these two cases the disk corona and jet were neglected $\left(A=0\right.$ and $\left.f_{\text {cor }}=0\right)$. The dotted line shows a modification of the stability curve by the corona $\left(f_{\text {cor }}=0.5\right)$. The solid line shows the effect of the jet $(A=0.05)$.

On the same plot (circles) we show the stability curve calculated similarly to the method adopted by Nayakshin et al. (2000), i.e. we computed the disk surface density from the vertically averaged disk structure as specified in that paper, but we assumed the standard viscosity law. For the Nayakshin et al. model the instability occurs for accretion rates as low as $\dot{m} \sim 0.03$. Therefore, in order to stabilize the disk at low $\dot{m}$ the authors had to postulate a strong corona. In our model we expect the instability to appear for accretion rates higher than $\dot{m} \sim 0.1$ at that radius and even higher than $\dot{m} \sim 0.2$ if half of the gravitational energy is dissipated in the corona $\left(f_{\text {cor }}=0.5\right)$.

\subsection{Time Evolution and Model Parame- ters}

We use the time evolution code originally developed and described in detail by Smak (1984) for the study of cataclysmic variables, and modified by Siemiginowska, Czerny \& Kostyunin (1996) in the context of ionization instability in AGN (see also Hameury et al. 1998).

We adopt the mass supply, $\dot{m}_{o}$, to the inner part of the disk as a model parameter and follow the time evolution of the disk under the radiation pressure instability in thermal and viscous timescale, as studied by Szuszkiewicz \& Miller (1998). The complete model is given by the mass supply rate, $\dot{m}_{o}$, viscosity parameter, $\alpha$, jet efficiency factor, $A$ (relating the fraction of energy carried by the jet to the local temporary accretion rate), and the (fixed) fraction of energy dissipated in the corona, $f_{\text {cor }}$. 


\section{RESULTS}

We calculate the time evolution of the disk for a set of parameters adequate to explain the timescales and the amplitudes of the typical outbursts observed in GRS 1915+105. In the following we assume that the black hole mass is equal to $M=10 M_{\odot}$ (estimated mass of the microquasar ranges from 7 to $33 M_{\odot}$ ), viscosity parameter $\alpha=0.01$ (as used by Nayakshin et al. 2000 on standard branch), $f_{\text {cor }}=0.5$ (moderate corona) and $A=0.05$ (mildly strong jet efficiency).

The instability can occur only above a certain accretion rate limit for a given mass and viscosity. For our choice of parameters, the disk is stable when the accretion rate $\dot{m}_{o}$ is smaller than 0.16 . For accretion rates above this limit the disk exhibits strong and regular outbursts.

The amplitude of the global outburst depends mostly on the shape of the stability curve in the innermost part of the disk, which constrains the jet efficiency $A$. The duration of the limit cycle, on the other hand, is basically determined by the viscous timescale at the outer radius of the instability zone in a stationary model, so it scales with the choice of the viscosity parameter $\alpha$.

In Figure 2 we show four lightcurves calculated for four values of the external accretion rate. Other parameters were kept the same. The plotted lightcurves represent the bolometric luminosity of the disk. However, they may be to some extent directly compared to the observed lightcurves expressed in counts/sec since the count rate in RXTE is strongly dominated by the soft energy band, determined mostly by the disk luminosity. The outbursts are almost periodic but their overall shape is significantly influenced by the mass supply rate. When $\dot{m}_{o}$ is only slightly larger than the minimum value required for instability to operate, the outbursts last only $\sim 100 \mathrm{~s}$ and the separation between them is relatively long. With an increase of $\dot{m}_{o}$ the duration of the bursts increases up to $\sim 1000$ s although the amplitude does not change strongly. Similar results were obtained by Nayakshin et al. (2000). Although their approach to the description of the disk structure was different from ours, it led to a similar stability curve and therefore outburst properties.

By comparing our model to the GRS $1915+105$ behavior we conclude that the observed shape of the microquasar's lightcurves can be obtained by varying the mass supply rate. The observed outburst's amplitude constrains the jet efficiency. The energy carried away in the jet varies during the cycle: it is negligible at low luminosity but can reach $\sim 15 \%-20 \%$ during the outburst, for assumed $\mathrm{A}=0.05$. The jet losses thus provide more efficient mechanism for stabilizing the disk than the advection and ensure that the luminosity of the source during outburst does not exceed the Eddington value.

The viscosity parameter $\alpha$ does not influence the shape of the stability curve, therefore the amplitude of the outburst remains the same as long as $\alpha$ is the same on the upper and lower branches. The change of $\alpha$ in this case results in the horizontal shift of the entire $\dot{M}-\Sigma$ curve. However, this strongly affects the surface density and viscous timescale, so for small $\alpha$ the surface density is higher and the viscous timescale is longer. The adopted value well represents the observed timescales in the microquasar.

\section{DISCUSSION}

We show that a physically viable instability due to radiation pressure in a standard disk model can well represent the time dependent behavior of GRS $1915+105$ if the advection and the presence of a moderate corona $\left(f_{\text {cor }}=0.5\right)$ and a jet is included in the model.

The mechanism operates for accretion rates above $\dot{m}_{o} \sim 0.16$ which is in agreement with the observations of GRS $1915+105$ - the source does not exhibit any outbursts if the mean luminosity temporarily drops below $2.1 \times 10^{38} \mathrm{erg} / \mathrm{s}$. The viscosity coefficient of order of 0.01 is appropriate to model the typical outburst duration as a function of the mass supply to the innermost part of accretion disk. The presence of the jet is necessary to explain the observed amplitude of the outburst, since without the jet the lightcurve variations are too large (factor $\sim 30$ ). The role of advection and the jet in the presented parameterization are similar, but for the required value of the jet efficiency the jet losses dominate and the effect of advection is never strong.

We conclude that the parameterization of the viscous stress as proportional to the total pressure well represents the true viscous stress properties 
and the radiation pressure instability is a promising model of the basic instability mechanism underlying the observed variability of this microquasar. The energy losses due to the jet are an essential ingredient of such model. Models based on modified viscosity law may allow for much lower jet efficiency. Observational constraints on the amount of energy carried by the jet will allow to distinguish between the two possibilities.

Acknowledgements. We are grateful to Marek A. Abramowicz for interesting discussions on the viscosity parametrization in advective discs. We thank Craig Markwardt for helpful discussion on the observed variability of GRS $1915+105$ and J.I. Smak for many discussions of the disk time evolution problems. We also thank the anonymous referee for suggestion on improvement our presentation. This work was supported in part by grants 2P03D01816 and 2P03D01519 of the Polish State Committee for Scientific Research. AS was supported by NASA Contract NAS8-39073 and NAG5-3391.

\section{REFERENCES}

Abramowicz, M. A., Czerny, B., Lasota, J.-P., Szuszkiewicz, E. 1988, ApJ, 332, 646

Belloni, T., Mendez, M., King, A. R., van der Klis, M., van Paradijs, J. 1997a, ApJ, 479, L145

Belloni, T., Mendez, M., King, A. R., van der Klis, M., van Paradijs, J. 1997b, ApJ, 488, L109

Belloni, T., Mendez, M., King, A. R., van der Klis, M., van Paradijs, J. 1997c, in Accretion Processes in Astrophysical Systems: Some Like It Hot!, College Park, MD, eds. S.S.Holt \& T.R. Kallman, AIP Conf. Proc., 431, p. 331

Castro-Tirado, A.J., Brandt, S., Lund, S. 1992, IAU Circ. 5590

Dörer, T., Riffert, H., Staubert, R., Ruder, H. 1996, A\&A, 311, 69

Feroci, M., Matt, G., Pooley, G., Costa, E., Tavani, M., Belloni, T., 1999, A\&A, 351, 985

Hameury, J.-M., Menou, K., Dubus, G., Lasota, J.-P., Hure, J.-M., 1998, MNRAS, 298, 1048 $422 \mathrm{c} 404$
Honma, F., Matsumoto, R., Kato, S. 1991, PASJ, 43,147

Lightman, A.P., Eardley, D.M. 1974, ApJ, 187, L1

Mirabel, I.F., Rodriguez, L. 1994, Nature, 371, 46

Muchotrzeb, B., \& Paczyński, B. 1982, Acta Astron., 32,1

Nayakshin, S., Rappaport, S., Melia, F. 2000, ApJ, 535, 798

Paczyński, B. \& Bisnovatyi-Kogan, G. 1981, Acta Asctron., 31, 283

Papaloizou, J.C.B., Lin, D.N.C., 1995, A.R.A.\&A., 33, 505

Pojmański, G. 1986, Acta Astron. 36, 69

Pringle, J.E., Rees, M.J., Pacholczyk, A.G. 1974, A.\&A., 29, 179

Różańska, A., Czerny, B., Życki, P.T., Pojmański, G. 1999, MNRAS 305, 481

Shakura, N.I., Sunyaev, R.A. 1973, A.\&A., 24, 337

Siemiginowska, A., Czerny, B., Kostyunin, V. 1996, ApJ, 458, 491

Smak, J. 1984, Acta Astron. 34, 161

Szuszkiewicz, E., Miller, J. 1998, MNRAS, 298, 888

Taam, R.E., Chen, X., Swank, J.H., 1997, ApJ, $485, \mathrm{~L} 83$

Trudolyubov, S.P., Churazov, E.M., Gilfanov, M.R. 1999, A\&A, 351, L18

This 2-column preprint was prepared with the AAS LATEX macros v5.0. 


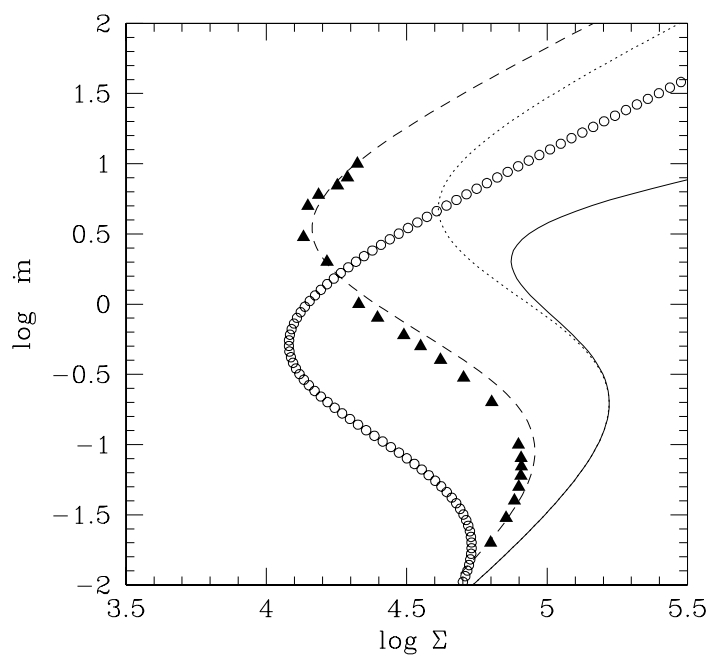

Fig. 1. - The stability curves calculated for $M=$ $10 M_{\odot}, \alpha=0.01$ and $R=10 R_{g}$. The triangles mark the solution with vertical structure model including advection and the circles mark the standard analytic solution. The dashed line is the solution with scaling adopted to match the vertical structure model. The dotted line marks the solution obtained from our analytic approximation with the corona included $\left(f_{c o r}=0.5\right)$, and the solid line shows the solution with the jet $(\mathrm{A}=0.05)$.
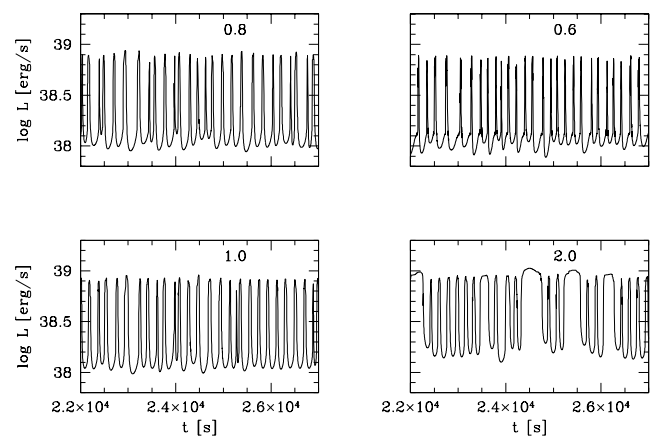

Fig. 2.- The lightcurves calculated for $M=$ $10 M_{\odot}, \alpha=0.01$. and four values of the external accretion rate: $0.6 \times 10^{-7}, 0.8 \times 10^{-7}, 1.0 \times 10^{-7}$ and $2.0 \times 10^{-7}\left[M_{\odot} / y r\right]$. The jet ejection described by Equation (1) is included with coefficient $A=0.05$ and the corona contribution is fixed at $f_{\text {cor }}=0.5$. 